\title{
Gallai-Colorings of Triples and 2-Factors of $\mathscr{B}_{3}$
}

\author{
Lynn Chua, ${ }^{1}$ András Gyárfás, ${ }^{2}$ and Chetak Hossain ${ }^{3}$ \\ ${ }^{1}$ Department of Mathematics, Massachusetts Institute of Technology, Cambridge, MA, USA \\ ${ }^{2}$ Alfréd Rényi Institute of Mathematics, Hungarian Academy of Sciences, P.O. Box 127, Budapest 1364, Hungary \\ ${ }^{3}$ Department of Mathematics, North Carolina State University, P.O. Box 8205, Raleigh, NC 27695, USA
}

Correspondence should be addressed to András Gyárfás; gyarfas.andras@renyi.mta.hu

Received 5 June 2013; Accepted 3 August 2013

Academic Editor: R. Yuster

Copyright (c) 2013 Lynn Chua et al. This is an open access article distributed under the Creative Commons Attribution License, which permits unrestricted use, distribution, and reproduction in any medium, provided the original work is properly cited.

\begin{abstract}
A coloring of the edges of the $r$-uniform complete hypergraph is a $G_{r}$-coloring if there is no rainbow simplex; that is, every set of $r+1$ vertices contains two edges of the same color. The notion extends $G_{2}$-colorings which are often called Gallai-colorings and originates from a seminal paper of Gallai. One well-known property of $G_{2}$-colorings is that at least one color class has a spanning tree. J. Lehel and the senior author observed that this property does not hold for $G_{r}$-colorings and proposed to study $f_{r}(n)$, the size of the largest monochromatic component which can be found in every $G_{r}$-coloring of $K_{n}^{r}$, the complete $r$-uniform hypergraph. The previous remark says that $f_{2}(n)=n$, and in this note, we address the case $r=3$. We prove that $\lceil(n+3) / 2\rceil \leq f_{3}(n) \leq\lceil 4 n / 5\rceil$, and this determines $f_{3}(n)$ for $n<7$. We also prove that $f_{3}(7)=6$ by excluding certain 2 -factors from the middle layer of the Boolean lattice on seven elements.
\end{abstract}

\section{Introduction}

Studying edge colorings of complete graphs without rainbow triangles (no triangles colored with distinct colors) originates from a famous paper of Gallai [1]; we will refer to such colorings as Gallai-colorings or $G_{2}$-colorings. A well-known property of $G_{2}$-colorings is that the edges of some color class span a connected subgraph containing all vertices. A natural extension of the concept is the $G_{r}$-coloring, of $K_{n}^{r}$, the complete $r$-uniform hypergraph on $n$ vertices. In a $G_{r}$-coloring the requirement is that no $K_{r+1}^{r}$ is colored with $r+1$ distinct colors; that is, there is no rainbow simplex. For $r \geq 3, G_{r}$-colorings of $K_{n}^{3}$ do not necessarily contain spanning connected color classes, but they must contain large ones. To define how large, let $f_{r}(n)$ be the size of the largest monochromatic component which can be found in every $G_{r}$-coloring of $K_{n}^{r}$. In terms of this function, $f_{2}(n)=n$, and in this note we address $f_{3}(n)$.

Theorem 1. $\lceil(n+3) / 2\rceil \leq f_{3}(n) \leq\lceil 4 n / 5\rceil$.

Proof. The upper bound (an unpublished note of the senior author and J. Lehel) follows from taking first the $G_{3}$-coloring of $K_{5}^{3}$ with five colors, where the vertex set is $\{1,2,3,4,5\}$, and the edges $i, i+1, i+2$ and $i, i+2, i+3$ are colored with $i$ (using (mod 5) arithmetic). This coloring can be "blown up" by replacing each vertex $i$ by a set $A_{i}$ so that the five sets have sizes that differ by at most one and defining the following straightforward extension of the original 5-coloring. Triples with vertices from three different $A_{i}$ are colored according to the color of the triple of their indices. Triples inside $A_{i}$ and triples in $A_{i} \cup A_{i+1}$ or in $A_{i} \cup A_{i+2}$ (with at least one vertex in each) are colored with color $i$.

The lower bound follows from the observation that every 4 -set must contain two 3 -sets of the same color. Thus the color of some 3 -set $T$ is repeated on another triple inside at least

$$
\frac{2\left(\begin{array}{l}
n \\
2
\end{array}\right)}{\left(\begin{array}{c}
n \\
3
\end{array}\right)}=\frac{n-3}{2}
$$

4 -sets containing $T$. This means that the monochromatic component containing $T$ has at least $(n-3) / 2+3$ vertices.

In fact, for odd $n>3$, the proof of the lower bound in Theorem 1 gives a condition for equality in terms of the bipartite graph $\mathscr{B}_{4,3}^{n}$ defined by the inclusions of the 4 - and 3-element 
sets of $\{1,2, \ldots, n\}$. A $(p, q)$-factor in a bipartite graph $[A, B]$ is a subgraph with

$$
d(v)= \begin{cases}p & \text { if } v \in A \\ q & \text { if } v \in B\end{cases}
$$

where $d(v)$ is the degree of vertex $v$.

Corollary 2. If the lower bound of Theorem 1 is sharp for some odd $n \geq 5$ then there is a $(2,(n-3) / 2)$-factor $F$ in $\mathscr{B}_{4,3}^{n}$, such that the union of the triples and quadruples in every component of $F$ has at most $(n+3) / 2$ elements.

Theorem 1 implies that $f_{3}(4)=f_{3}(5)=4$ and $f_{3}(6)=5$ but gives only $f_{3}(7) \in\{5,6\}$. Notice that, for $n=5$, the upper bound construction of Theorem 1 is a $(2,1)$-factor in $\mathscr{B}_{4,3}^{5}$ required in Corollary 2, consisting of the shifts of the 3vertex component $1234,123,134$ modulo 5 . Is it possible that for some $n>5$ there is a $(2,(n-3) / 2)$-factor providing a better upper bound for $f_{3}(n)$ ? The first case to consider is $\mathscr{B}_{3}=$ $\mathscr{B}_{4,3}^{7}$, the "middle-layer graph" in the boolean lattice on seven elements. To decide whether $f_{3}(7)=5$, we will determine all possible 2 -factors required by Corollary 2. In Lemma 3 we show that these are 2 -factors in $\mathscr{B}_{3}$ whose components are 6-, 8 -, or 10-cycles of the following type which we call a $\mathscr{C}$-factor:

(i) $C_{6}=C_{6}(a, b ; c, d, e)$ the 6-cycle $a b c, a b c d, a b d, a b d e$, abe, $a b c e, a b c$,

(ii) $C_{8}=C_{8}(a ; b c d e)$ the 8-cycle $a b c, a b c d$, acd, acde, ade, adeb, aeb, aebc, $a b c$,

(iii) $C_{10}=C_{10}(a b c d e)$ the 10-cycle $a b c, a b c d, b c d, b c d e$, $c d e, c d e a, d e a, d e a b, e a b, e a b c, a b c$,

where $\{a, b, c, d, e\} \subset\{1,2, \ldots, 7\}$. It is worth mentioning that the cycles of a $\mathscr{C}$-factor can be considered as subdivisions of certain triangles, quadrangles, and pentagons of the Johnson graph $J(7,3)$, where $J(n, 3)$ (sometimes referred to as the tetrahedral graph) is the graph whose vertices are the triples of an $n$-element set and edges are between triples intersecting in two vertices. The speciality of these cycles is that the triples (and quadruples) are among five elements $(a, b, c, d$, and $e$ ). The 2 -factors of the middle layer graph are investigated before, primarily because of the middle level (or revolving door) conjecture; see [2] and its references.

Lemma 3. $f_{3}(7)=5$ if and only if $\mathscr{B}_{3}$ has a $\mathscr{C}$-factor.

Proof. If $\mathscr{B}_{3}$ has a $\mathscr{C}$-factor, we have a $G_{3}$-coloring of $K_{5}^{3}$ obtained by coloring the 3 -sets with the same color if and only if they are in the same cycle in the $\mathscr{C}$-factor. The triples of each cycle determine a monochromatic component with five vertices; hence $f_{3}(7) \leq 5$, and together with the lower bound of Theorem 1, we get $f_{3}(7)=5$.

Conversely, suppose that $f_{3}(7)=5$, and consider the $G_{3}$ coloring of $K_{7}^{3}$ with all monochromatic components having at most five vertices. Let $B \subset \mathscr{B}_{3}$ be the bipartite graph defined by selecting two 3 -sets of the same color from each 4-element subset of $\{1,2, \ldots, 7\}$. The graph $B$ must be 2 -regular, otherwise some 3 -set, say in color red, would be selected into at least three 4 -sets, resulting in a red component of at least six vertices. Thus $B$ is a 2 -factor in $\mathscr{B}_{3}$, and its components are cycles. But $\mathscr{B}_{3}$ contains no $C_{4}$ since two 4 -sets can share at most one 3-set. Also, $\mathscr{B}_{3}$ cannot have a $C_{n}$ component with $n \geq 12$; otherwise the corresponding coloring of $K_{5}^{3}$ has a monochromatic component with more than five vertices, since there are only five 4-sets on five elements. Thus the components of $B$ are elements of $\left\{C_{6}, C_{8}, C_{10}\right\}$. However, these cycles have to form subdivisions of $\left\{C_{3}, C_{4}, C_{5}\right\}$ in the Johnson graph $J(5,3)$, such that the union of triples on the edges of a cycle forms different 4-sets. This means that the edges of $C \in\left\{C_{3}, C_{4}, C_{5}\right\}$ which are to be subdivided must be in different main cliques of $J(5,3)$, where a main clique $K_{4}$ is the complete subgraph of $J(5,3)$ determined by the triples of $\{1,2,3,4,5\}-\{i\}$ for some $i \in\{1,2,3,4,5\}$. The edge set of $J(5,3)$ is partitioned into five main cliques, and we can label the edges in each main clique by $i$, such that $C$ consists of edges with different labels. If two edges $i, j$ meet, then the corresponding 3 -set is $\{1,2,3,4,5\}-\{i, j\}$. For a $C_{3}$ with edges labeled 3, 4, and 5, the corresponding 3 -sets are 123, 124, and 125 . One can similarly check for $C_{4}$ and $C_{5}$ that the only possibilities are the cycles in the definition of the $\mathscr{C}$-factor.

In Sections 2 and 3 we prove the following.

Theorem 4. $\mathscr{B}_{3}$ has no $\mathscr{C}$-factor.

Lemma 3 and Theorems 1 and 4 give the following.

Corollary 5. $f_{3}(7)=6$.

Theorem 4 might be true in a stronger form.

Question 1. Is there a 2-factor in $\mathscr{B}_{3}$ in which all cycles have length of at most 10 ?

It is worth mentioning that, in the family of 2-factors constructed in [2], all components are cycles with length divisible by 14; thus it does not answer Question 1.

The proof of Theorem 4 is based on properties of graphs shown in Figure 2. They are the graphs on seven vertices having 11,10 , or 9 edges with the property that every set of three vertices contains at least one edge (complements of trianglefree graphs that are close to the extremal Turán graphs). We need to analyze star-partitions $x S_{3}+y S_{4}+z S_{5}$ of $K_{7}^{3}$, where the star $S_{i}$ is the hypergraph having $i$ triples containing two fixed vertices, called the base. Note that $K_{7}^{3}$ is an interesting object to decompose. For example, $K_{7}^{3}$ can be partitioned into 5 "tight cycles" (consecutive triples in a cyclic permutation of $V\left(K_{7}^{3}\right)$ ) but cannot be partitioned into 5 Fano planes. In fact, $K_{7}^{3}$ does not contain three edge disjoint Fano planes (Cayley [3]). However, $K_{7}^{3}$ can be partitioned into 6 linear hypergraphs (but not into 5) [4].

Decompositions of complete hypergraphs into stars (hyperclaws) have been investigated $[5,6]$, and this is strongly related to our subject. In particular, the following decomposition problem is related to the case $n=9$. Let $H$ be a star $S_{4}$ with vertex set $x_{1}, \ldots, x_{6}$ and with base $x_{1} x_{2}$. Define the mate of $H$ as the 4-uniform hypergraph with the six 4-element subsets $\left\{x_{1}, x_{2}, x_{i}, x_{j}\right\}$ where $i, j, i \neq j$ runs over the unordered pairs of $3,4,5,6$. 

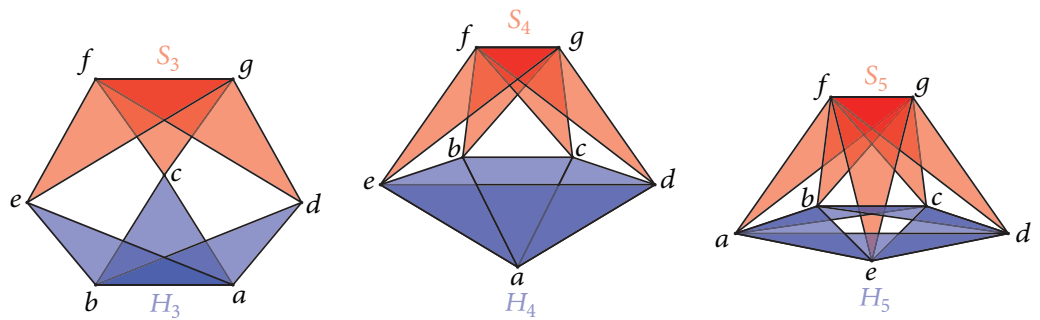

FIGURE 1: Diagram of the stars $S_{i}$ with base $f g$ (red) and the corresponding hypergraphs $H_{i}$ (blue).

Question 2. Is it possible to partition $K_{9}^{3}$ into stars $S_{4}$ so that their mates partition $K_{9}^{4}$ ? (Star partition without the mate condition is possible [5].)

In fact, Question 2 is a very special case of the $\mathscr{C}$-factor problem arising from the question whether $f_{3}(9)>6$. Here $\mathscr{C}$ should be the list of components of a $(2,3)$-factor of $\mathscr{B}_{4,3}^{9}$ that contain triples and quadruples of six elements (out of nine). Such components arise by subdividing the edges of several 3regular subgraphs of $J(6,3)$. Question 2 came about by considering the component of a $(2,3)$-factor from the subdivision of a $K_{4} \in J(6,3)$. However, other components come from the subdivision of the following graphs: $K_{2} \times K_{3}$, the graph obtained from $K_{2} \times K_{3}$ by subdividing two edges not in a triangle and connecting the subdivision points with an edge, the cube, and the Petersen graph-we checked with a computer that this list is exhaustive. For example, the cube in $J(6,3)$ represented by

$$
123,124,245,235,136,146,456,356
$$

and the Petersen graph in $J(6,3)$ represented by

$$
123,124,235,136,145,345,156,246,256,346
$$

generate components with 8 and 10 triples (and 12 and 15 quadruples) in $\mathscr{B}_{4,3}^{9}$. Thus there are five possible components of a $(2,3)$-factor in $\mathscr{C}$, and this probably makes it difficult to decide whether $f_{3}(9)>6$.

\section{Special 2-Factors in $\mathscr{B}_{3}$ and Star Decompositions of $K_{7}^{3}$}

We use $\mathscr{C}_{6}, \mathscr{C}_{8}$, and $\mathscr{C}_{10}$ to denote the set of possible cycles of a $\mathscr{C}$-factor with different parameter choices from $\{1,2, \ldots, 7\}$ (e.g., $\left.\left|\mathscr{C}_{10}\right|=4 !\left(\begin{array}{l}7 \\ 5\end{array}\right)\right)$. Then $\mathscr{C}=\mathscr{C}_{6} \cup \mathscr{C}_{8} \cup \mathscr{C}_{10}$, and a $\mathscr{C}$-factor is a 2 -factor of $\mathscr{B}_{3}$ such that each cycle in it belongs to $\mathscr{C}$.

The type of a $\mathscr{C}$-factor is the vector $(x, y, z)$ where $x, y$, and $z$ denote the number of cycles of $\mathscr{C}$ from $\mathscr{C}_{6}, \mathscr{C}_{8}$, and $\mathscr{C}_{10}$, respectively. One can easily determine the possible types of a $\mathscr{C}$-factor by determining the nonnegative integer solutions of $35=3 x+4 y+5 z$. There are 14 types, and we will eliminate all of them.

First we reformulate the existence of a $\mathscr{C}$-factor as a decomposition problem of $K_{7}^{3}$. We define $S_{i}$, the $i$-star, as a 3uniform hypergraph having $i$ edges containing a fixed pair of vertices, called the base of the star. A star partition of $K_{n}^{3}$ is a partition of all edges into stars; in particular $K_{n}^{3}=\sum_{i=1}^{k} x_{i} S_{i}$ means that $K_{n}^{3}$ is partitioned into $x_{i}$ copies of $S_{i}$, for $i=$ $1,2, \ldots, k$. Notice that the complements of the four-element sets in the cycles of a $\mathscr{C}$-factor provide a starpartition of $K_{7}^{3}$. On the other hand, the three-element sets in the cycles of a $\mathscr{C}$-factor also give a partition of $K_{7}^{3}$ into copies of the hypergraphs $\mathrm{H}_{3}, \mathrm{H}_{4}$, and $\mathrm{H}_{5}$ which we call the complementary partition $\left(H_{3}\right.$ is isomorphic to $S_{3}, H_{4}$ has four triples containing a fixed vertex and consecutive pairs on a 4-cycle of pairs, and $\mathrm{H}_{5}$ has five consecutive triples on a 5-cycle of pairs; these are illustrated in Figure 1). These considerations lead to the following.

Proposition 6. $\mathscr{B}_{3}$ has a $\mathscr{C}$-factor of type $(x, y, z)$ if and only if $K_{7}^{3}$ has a star partition $x S_{3}+y S_{4}+z S_{5}$ together with the complementary partition $x \mathrm{H}_{3}+y \mathrm{H}_{4}+z \mathrm{H}_{5}$.

2.1. Decomposing $K_{7}^{3}$ along Turán Graphs. We associate a weighted graph $G$, the base graph, with a star partition $x S_{3}+$ $y S_{4}+z S_{5}$ on $K_{7}^{3}$ as follows. The vertex set of $G$ is $\{1,2, \ldots, 7\}$, and the $(x+y+z)$ edges are determined by the pairs of the bases of the stars in the partition. The graph obtained this way has no multiple edges because different stars must have different bases, since two stars have at least six edges. The weight of an edge $e$ is $i$ if $e$ is the base of an $S_{i}$.

If $G$ is the base graph of a star partition $x S_{3}+y S_{4}+z S_{5}$ on $K_{7}^{3}$, then every triple of $\{1,2, \ldots, 7\}$ contains at least one edge of $G$. By Turán's theorem (complementary form), $x+y+z \geq 9$, and for $x+y+z=9, x+y+z=10$, the graphs $G=T_{9}$ or $G=$ $T_{10}$ are unique: the vertex disjoint union of $K_{3}$ and $K_{4}$, with an edge in between in the case of $T_{10}$.

When $x+y+z=11$, easy inspection reveals five possibilities for the base graph. Let $T_{11}^{1}$ be the disjoint union of $K_{5}$ and $K_{2}$. Then $T_{11}^{2}, T_{11}^{3}$, and $T_{11}^{4}$ are disjoint unions of $K_{3}$ and $K_{4}$ plus two edges between them, with a common vertex in $K_{4}$, a common vertex in $K_{3}$, and no common vertex, respectively (see Figure 2). Finally, $T_{11}^{5}$ is the disjoint union of two $K_{3}$-s plus one edge between them plus four edges from the seventh vertex to the vertices of degree two (complement of $K_{3,3}$ with a subdivided edge).

These considerations eliminate the vectors of types $(0,0,7),(2,1,5),(1,3,4)$, and $(0,5,3)$ and reduce the problem to the following cases. 

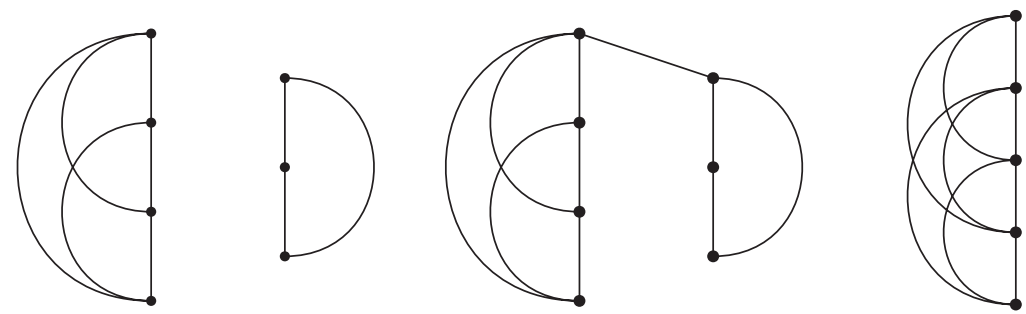

$T_{9}$

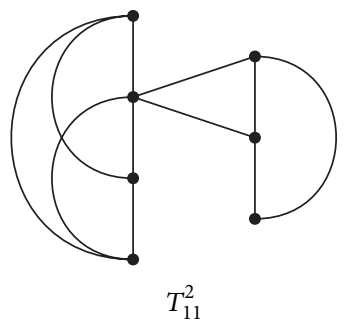

$T_{10}$
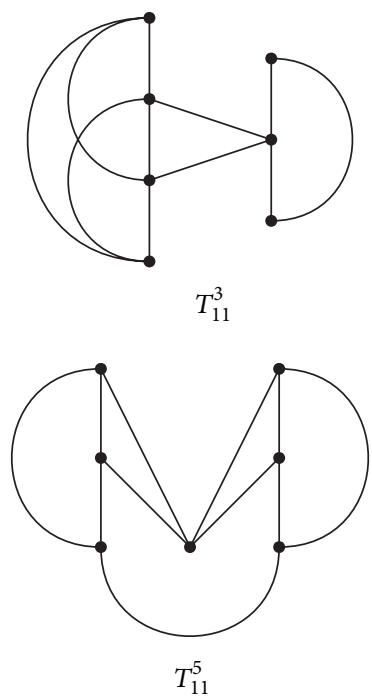

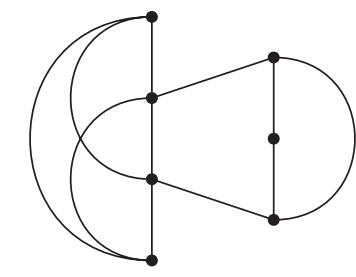

$T_{11}^{4}$

FIgURE 2: Diagram of the possible base graphs $T_{9}, T_{10}, T_{11}^{1}, \ldots, T_{11}^{5}$.

Lemma 7. Suppose that $\mathscr{B}_{3}$ has a $\mathscr{C}$-factor of type $(x, y, z)$. Then one has on $K_{7}^{3}$ a star partition with base graph $G$ together with a complementary partition with the following parameters.

(i) $G=T_{9}$ and $(x, y, z) \in\{(1,8,0),(5,0,4),(4,2,3)$, $(3,4,2),(2,6,1)\}$.

(ii) $G=T_{10}$ and $(x, y, z) \in\{(5,5,0),(7,1,2),(6,3,1)\}$.

(iii) $G \in\left\{T_{11}^{i}: i \in\{1,2,3,4,5\}\right\}$ and $(x, y, z) \in\{(9,2,0)$, $(10,0,1)\}$.

The following lemma will eliminate six types of vectors.

Lemma 8. Suppose that $\mathscr{B}_{3}$ has a $\mathscr{C}$-factor of type $(x, y, z)$ with $z>0$. Then the base graph has a 5-cycle.

Proof. Suppose that $\mathscr{B}_{3}$ has a $C_{10}$ cycle, $C=C_{10}(12345)$, the 10 -cycle $123,1234,234,2345,345,3451,451,4512,512,5123$, and 123 , which generates an $S_{5}$ with base edge 67. Consider the remaining five 3-sets formed from the vertices 12345 which are not in $C: 124,134,135,235$, and 245 . Observe that 124 is only contained in the 4 -sets $1234,1245,1246$, and 1247 . Since 1234,1245 are in $C$, then 124 must be in the same cycle as 1246 and 1247. Hence we have a cycle on the vertices 12467. Similarly, we have four other cycles on $13467,13567,23567$, and 24567. The base edges of the five stars generated by these five cycles are, respectively, $35,25,24,14,13$, which form a 5-cycle in the base graph.
Since $T_{9}, T_{10}$, and $T_{11}^{2}$ do not contain 5-cycles, we have the following.

Lemma 9. Suppose that $\mathscr{B}_{3}$ has a $\mathscr{C}$-factor of type $(x, y, z)$. Then one has on $K_{7}^{3}$ a star partition with base graph $G$ together with a complementary partition with the following parameters.

(i) $G=T_{9}$ and $(x, y, z)=(1,8,0)$.

(ii) $G=T_{10}$ and $(x, y, z)=(5,5,0)$.

(iii) $G \in\left\{T_{11}^{i}: i \in\{1,2,3,4,5\}\right\}$ and $(x, y, z) \in\{(9,2,0)$, $(10,0,1)\}$.

\section{Eliminating the Remaining Types}

3.1. $(10,0,1)$. Following the proof of Lemma 8 , we can label the 10 -cycle as $C_{10}(12345)$, and we have 6 -cycles on 12467 , $13467,13567,23567$, and 24567 . In a 6-cycle, two vertices are repeated in every element. Since the five 6-cycles above contain, respectively, the 3 -sets $124,134,135,235$, and 245, the two repeated vertices must be from these 3 -sets. In particular, the 3 -sets $167,267,367,467$, and 567 are not contained in any of these 6-cycles. There are ten 4-sets containing these five 3sets. But each of the five 6-cycles contains one of these 4-sets. Hence there are five remaining 4-sets which contain five 3sets, and the only possibility is that these ten elements form a 10 -cycle. Then there are two 10-cycles, a contradiction. 
3.2. $(1,8,0)$. Label $G=T_{9}$ so that $\{1,2,3,4\}$ spans a $K_{4}$ and $\{5,6,7\}$ spans a $K_{3}$.

One of the edges of the $K_{3}$ must generate the triple 567; say $e=56 . e$ must also generate the triples $156,256,356$, and 456 . But $e$ can generate at most 4 triples, a contradiction.

3.3. (5,5,0). Label $G=T_{10}$ so that $\{1,2,3,4\}$ spans a $K_{4}$, $\{5,6,7\}$ spans a $K_{3}$, and the tenth edge is $(4,5)$.

(i) The edge $e=67$ of $G$ must be the base of a copy $P_{1}$ of $S_{4}$ since the triples $i, 6,7$ for $i \in[4]$ must be based on $e$. Let (w.l.o.g) 1, 2, 3, 4 be the cyclic ordering of the 4-cycle in the copy of $H_{4}$ complementary to $P_{1}$ (the triples in $\left.C_{8}(5 ; 1234)\right)$.

(ii) Consider the four base edges of $G$ on the 4-cycle $U=$ $1,2,3,4,1$. We claim that all of them have weight 4 . Suppose this is not the case, and $f=i j$ is covered by a star $P_{2}$ in $S_{3}$ where $i, j$ are consecutive on $U$. Observe that the triples $i, j, 6$ and $i, j, 7$ must be in $P_{2}$. Therefore the triple $M=\{1,2, \ldots, 7\} \backslash\{i, j, 6,7\}$ is covered by the copy of $\mathrm{H}_{3}$ complementary to $P_{2}$. However, $M=$ $\{5, i+2, j+2\}$ with $(\bmod 4)$, addition thus it is also covered by the copy of $H_{4}$ complementary to $P_{1}$, a contradiction.

(iii) At this point, we know that the five base edges are of weight 4 , and the rest of the edges have weight 3 . In particular, 13 and 57 have weight 3 , and both uniquely determine their stars in $S_{3}$, namely, 135, 136, 137 and $571,572,573$. But then the triple 246 is doubly covered by their complementary copies of $\mathrm{H}_{3}$-s, a contradiction, finishing the proof.

3.4. $(9,2,0)$. The base graph $T_{11}^{1}$ cannot occur since the base edge $K_{2}$ must generate 5 triples.

Case 1. Base graph is $T_{11}^{5}$, the union of edge 12 , the triangles $1,3,4$ and 2,5,6, and the star 37, 47, 57, 67 .

The base edges $e=34$ and $f=56$ must generate the triples $234,345,346$ and $156,356,456$, respectively. They cannot both generate $S_{3}$-s because then the triple 127 would be doubly covered by their complementary $\mathrm{H}_{3}$-s.

One of $e, f$, say $e=34$ generates an $S_{4}$. Suppose first that $f$ generates an $S_{3}$. Then 13 and 14 both generate $S_{4}$-s; otherwise 247 or 237 would be doubly covered by their complementary $\mathrm{H}_{3}$-s. But this is a contradiction, having at least three $\mathrm{S}_{4}$-s in the star partition. Thus $f$ also generates an $S_{4}$. Consequently, 25 and 26 both generate $S_{3}$-s and their complementary $\mathrm{H}_{3}$-s contain the triples 167 and 157, respectively. However, at least one of these triples is also covered by the complementary $\mathrm{H}_{4}$ of the $S_{4}$ generated by $e$, a contradiction.

Case 2. Base graph is $T_{11}^{4}$, labeled so that $\{1,2,3,4\}$ spans a $K_{4}$ and $\{5,6,7\}$ spans a $K_{3}$, with two additional edges $(4,5)(1,7)$.

The base edge $e=23$ must generate the triples 235, 236, and 237. Suppose that $e$ generates an $S_{3}$; then the complementary $\mathrm{H}_{3}$ contains the triples 145,146 , and 147. This implies that the base edges 56, 57, and 67 must all generate $S_{4}$-s. Otherwise, suppose that 56 generates an $S_{3}$. Since 56 must generate the triples 256 and 356 , the triple 147 is in the complementary $\mathrm{H}_{3}$, and it is doubly covered by the complementary $\mathrm{H}_{3}$ of $e$. Similarly, if 57 and 67 generate $S_{3}$-s, then the triples 146 and 145 would be doubly covered. Thus $e$ must generate an $S_{4}$.

Consider the complementary $\mathrm{H}_{4}$ generated by $e$, w.l.o.g. let it be $C_{8}(1 ; 4567)$. Then it contains at least two of the triples 145,146 , and 147 , a contradiction.

Case 3. Base graph is $T_{11}^{3}$, labeled so that $\{1,2,3,4\}$ spans a $K_{4}$ and $\{5,6,7\}$ spans a $K_{3}$, with two additional edges $(1,5)$ and $(4,5)$.

The base edge $e=67$ must generate the triples 167, 267, 367 , and 467 and must thus generate an $S_{4}$. Consider the base edges 23,56 , and 57.23 must generate the triples 235,236 , and 237; 56 must generate 526, 536; 57 must generate 527 and 537. If 23 generates an $S_{3}$, then 56,57 must both generate $S_{4}$-s, otherwise the triples 147, 146 would be doubly covered. But then there would be three $S_{4}$-s. Hence 23 generates an $S_{4}$, and all the remaining edges generate $S_{3}$-s.

Consider the base edges 12,13,14, 24, and 34. Since each of these generates two triples with 6 and 7, their complementary $\mathrm{H}_{3}$-s contain the triples $345,245,235,135$, and 125 , respectively. However, the complementary $H_{4}$ of 67 , which w.l.o.g. can be written as either $C_{8}(5 ; 1234)$ or $C_{8}(5 ; 1243)$ must contain at least three of these triples, a contradiction.

Case 4. Base graph is $T_{11}^{2}$, labeled such that $\{1,2,3,4\}$ spans a $K_{4}$ and $\{5,6,7\}$ spans a $K_{3}$, with two additional edges $(4,5)$, $(4,7)$.

The base edge 56 must generate the triples $156,256,356$, and the base edge 23 must generate 235, 236, and 237. If both of them generate $\mathrm{S}_{3}$-s, then the complementary $\mathrm{H}_{3}$-s would doubly cover 147. Hence at least one of 56 or 23 must generate an $S_{4}$. Similarly, if 67,13 both generate $S_{3}$-s, then 245 would be doubly covered by their complementary $\mathrm{H}_{3}$-s, and if 57,12 both generate $S_{3}$-s, then 346 would be doubly covered. Then we have at least three $S_{4}$-s, a contradiction.

\section{Acknowledgment}

The authors would like to acknowledge the support of the Elective Undergraduate Research Program of the Budapest Semesters in Mathematics, 2013 Spring.

\section{References}

[1] T. Gallai, “Transitiv orientierbare Graphen,” Acta Mathematica Hungarica, vol. 18, pp. 25-66, 1967, English translation by F. Maffray and M. Preissmann, in Perfect Graphs, J. L. RamirezAlfonsin and B. A. Reed, Eds., pp. 25-66, John Wiley and Sons, New York, NY, USA, 2001.

[2] T. Mütze and F. Weber, "Construction of 2-factors in the middle layer of the discrete cube," Journal of Combinatorial Theory A, vol. 119, no. 8, pp. 1832-1855, 2012.

[3] A. Cayley, "On the triadic arrangements of seven and fifteen things," The London, Edinburgh and Dublin Philosophical Magazine and Journal of Science, vol. 37, pp. 50-53, 1850.

[4] M. Axenovich, A. Gyárfás, H. Liu, and D. Mubayi, "Multicolor Ramsey numbers for triple systems," http://arxiv.org/abs/1302 .5304 . 
[5] A.-F. Mouyart, "Decomposition of the complete hypergraph into hyperclaws," Discrete Mathematics, vol. 76, no. 2, pp. 137150, 1989.

[6] S. Yamamoto and S. Tazawa, "Hyperclaw decomposition of complete hypergraphs," Annals of Discrete Mathematics, vol. 6, pp. 385-391, 1980. 


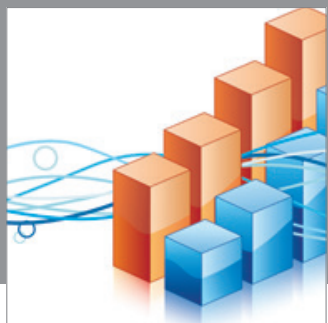

Advances in

Operations Research

mansans

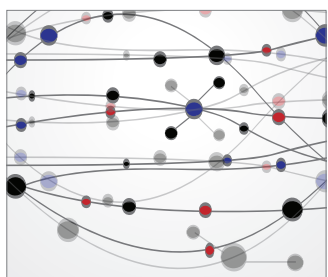

The Scientific World Journal
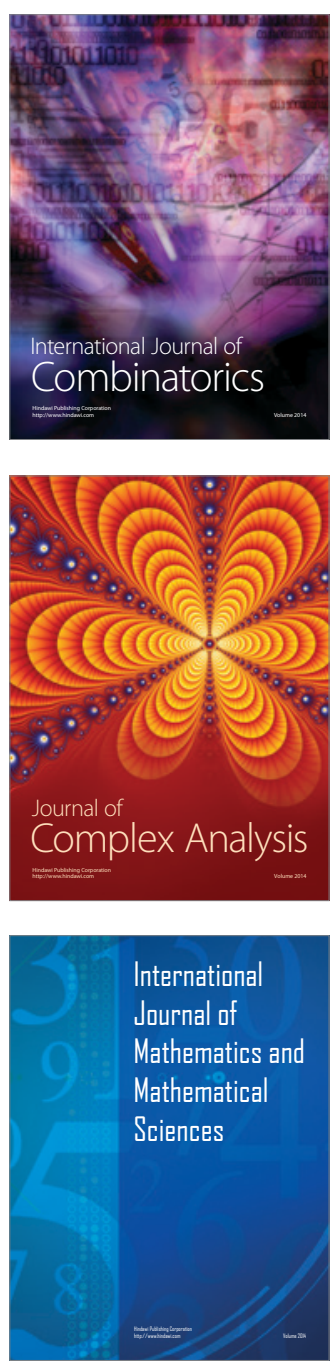
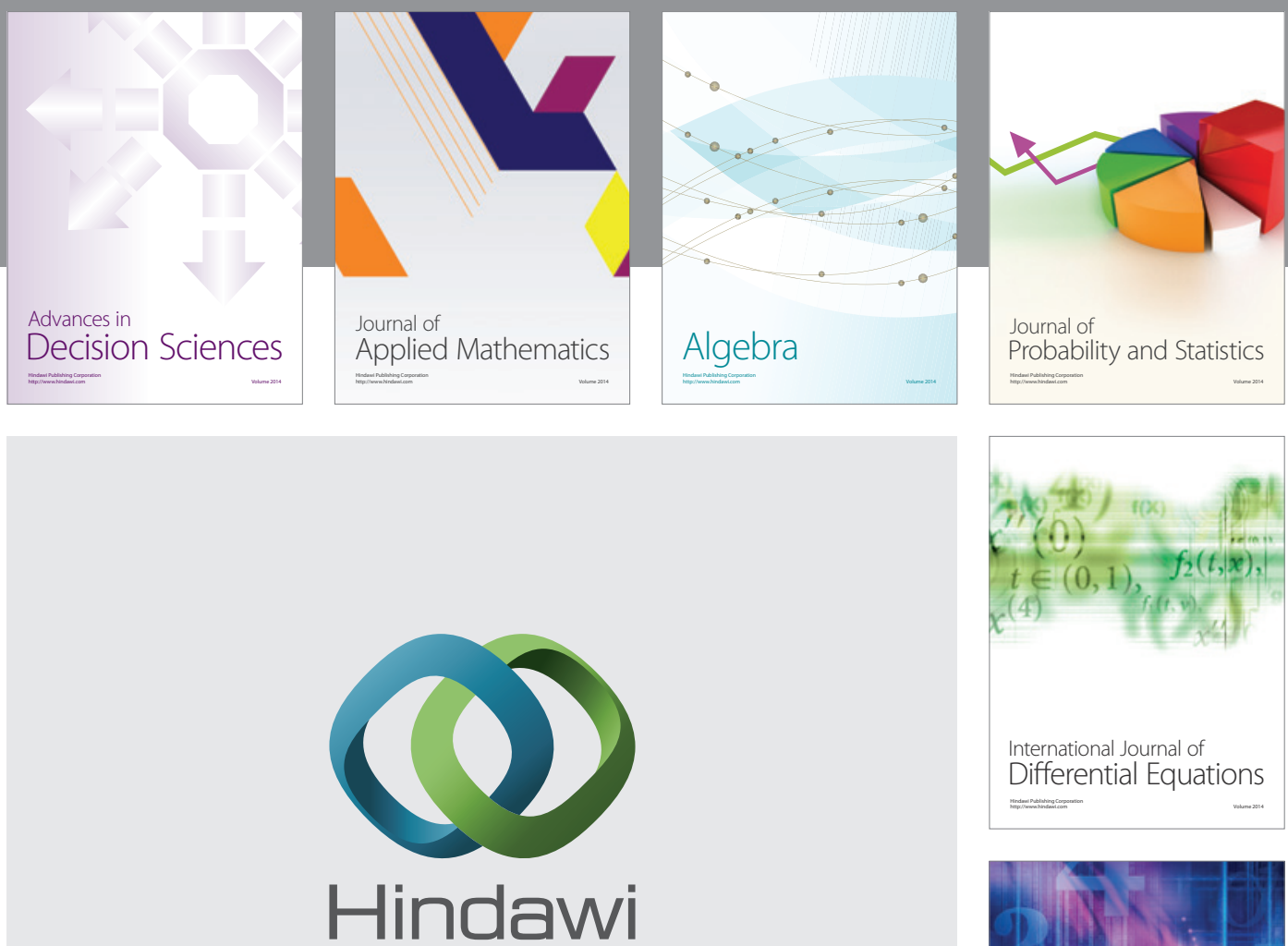

Submit your manuscripts at http://www.hindawi.com
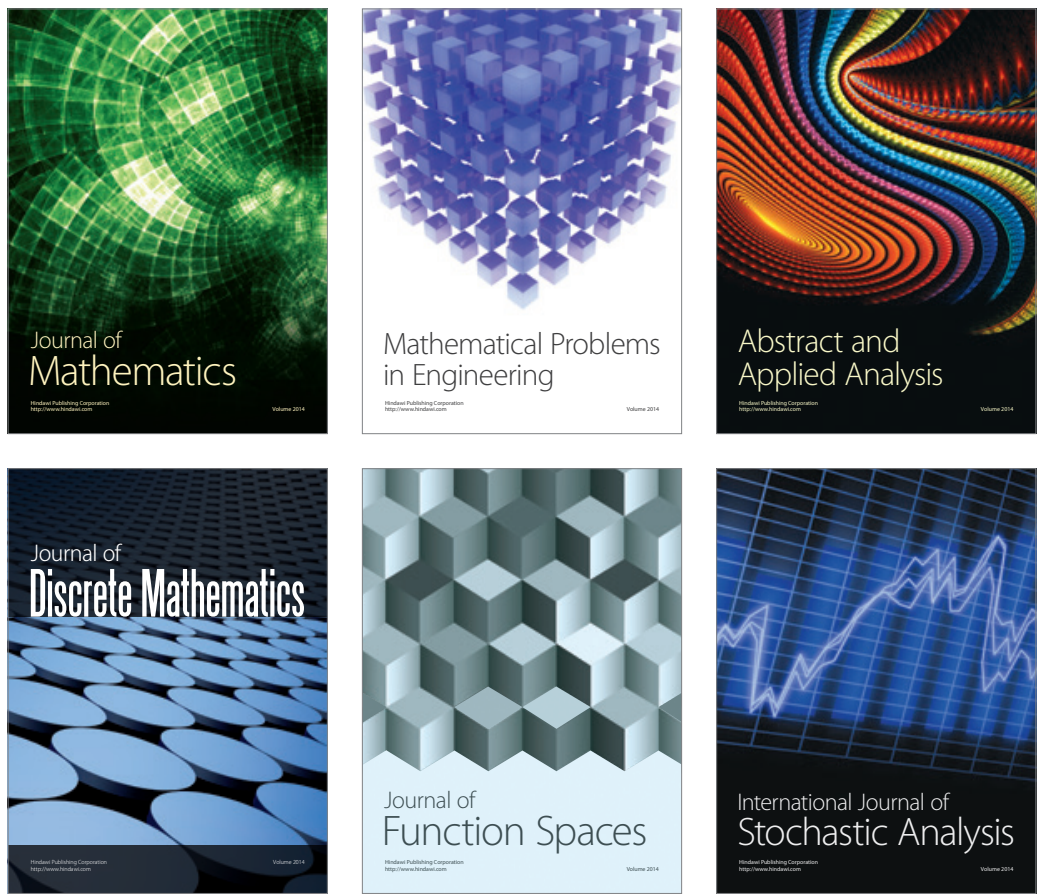

Journal of

Function Spaces

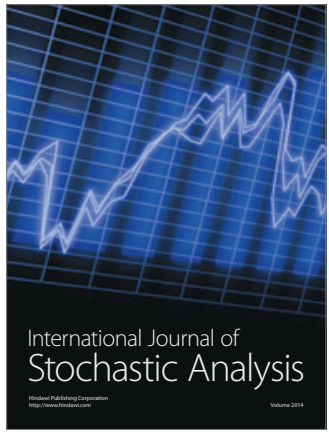

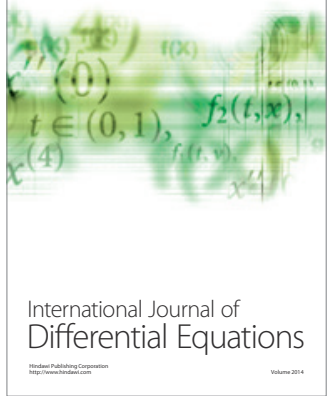
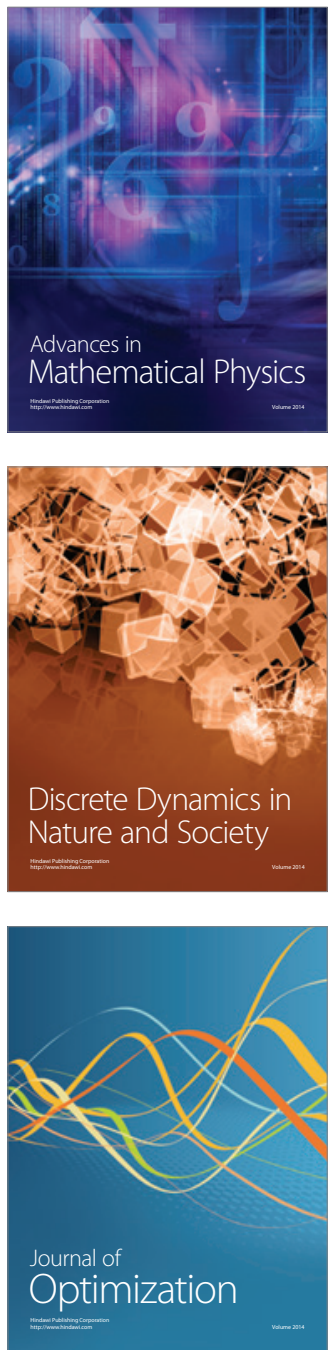